\title{
So unzuverlässig ist die Dermatoskopie
}

\author{
Viele der beim Hautkrebsscreening eingesetzten Kriterien für die \\ Dermatoskopie sind offenbar nur begrenzt aussagekräftig und werden von \\ den Untersuchern zudem ganz unterschiedlich bewertet. Forscher aus den \\ USA fordern bessere, einheitliche Algorithmen.
}

B isher ist es nicht gelungen, einheitliche und verlässliche Kriterien für die Dermatoskopie festzuschreiben, nach denen man zwischen melanozytären und nicht melanozytären Läsionen differenzieren kann. Wie unterschiedlich dermatoskopische Kriterien selbst innerhalb einer Gruppe von erfahrenen Untersuchern gehandhabt werden und wie unterschiedlich auch die Aussagekraft der verwendeten Algorithmen ist, haben Hautärzte aus den USA gezeigt.

An ihrer Internet-basierten Studie nahmen 130 Untersucher teil; im Schnitt hatten diese zwölf Jahre dermatologische Berufserfahrung. Die Teilnehmer sollten Fotos von 477 Hautläsionen beurteilen. In 119 Fällen handelte es sich um Melanome. Die Untersucher bekamen erst eine Nahaufnahme der Hautläsion zu sehen, dann wurde ihnen das Dermatoskopiebild präsentiert. Im Anschluss daran sollten sie angeben, welche Kriterien aus einer umfassenden Liste, zusammengetragen aus den gängigen Algorithmen (ABCD-Regel, CASH, Regel nach Menzies, 7- bzw. 3-Punkte-Checkliste sowie „chaos and clues“), für die Läsion zutrafen und welche nicht. Die Liste enthielt sowohl etablierte Kriterien (z. B. Muster, Farbe, Farb- und Muster-Symmetrie, Kantenschärfe) als auch erst kürzlich identifizierte Kriterien mit hoher Spezifität für Melanome (z. B. negatives Pigmentnetz, Chrysalis-Strukturen, polymorphe Gefäße).

Am Ende der Studie lagen 5.670 Einzelauswertungen vor. Bei den Kriterien atypisches Netzwerk, blau-weißer Schleier, Regression und atypische Gefäße stimmten die Teilnehmer in ihrer Beurteilung sehr wenig bis wenig überein, mit einer Intra-Klassen-Korrelation (ICC) zwischen 0,01 und 0,4 (1 steht für perfekte Übereinstimmung). Mittlere Übereinstimmung wurde für Kommagefäße, Abwesenheit von Gefäßen, dunkelbraune Farbe und strukturelle Unregelmäßigkeit gefunden (ICC 0,4 bis 0,6).
Am stärksten mit der Diagnose eines Melanoms assoziiert waren ausgeprägte Strukturunregelmäßigkeit (OR 6,6), Muster-Asymmetrie (OR 4,9), nicht organisiertes Muster (OR 3,3), ein Randscore von 5 oder 6 (OR 3,1 bzw. 3,3) und Asymmetrie der Konturen (OR 3,2). Für alle diese ebenso wie für atypische $\mathrm{Ge}$ fäße und polymorphe Gefäße bestand ein signifikanter Zusammenhang.

Dagegen sprachen Kommagefäße (OR $0,4)$, peripheres Netz mit zentraler Hyperpigmentation, globuläres Muster, symmetrisches Muster aus zwei Komponenten, regelmäßige braune Punkte oder Globuli, Abwesenheit von Gefäßen (OR jeweils 0,5); regelmäßiger Farbfleck (OR $0,4)$ und hellbraune Farbe (OR 0,6) eher für einen Nävus. Unter den neu identifizierten Kriterien waren negatives Pigmentnetz (OR 1,4) und weiße glänzende Strukturen (OR 2,5) signifikant mit dem Melanomstatus assoziiert. Bei beiden stimmten die Untersucher jedoch sehr wenig überein.

Im direkten Vergleich der Algorithmen hatte die Menzie-Regel die höchste Sensitivität für die Erkennung eines Melanoms (95,1\%), die 3-Punkte-Checkliste die geringste $(68,9 \%)$. Bei der Spezifität lag die ABCD-Regel vorne (59,4\%), Menzie (24,8\%) war Schlusslicht.

Fazit: Die Ergebnisse bestätigen den Bedarf nach verbesserten Kriterien und Algorithmen, so die Autoren. Diese müssten leicht zu lernen, valide und verlässlich sein. Vor allem sei ein einheitlicher Algorithmus für die Dermatoskopie gefordert. Hierfür müssten die bestehenden Kriterien aber nicht aufgegeben werden; es gehe vielmehr darum, das bestehende Armamentarium in Studien zu schärfen und die Untersucher besser zu schulen. Dr. Elke Oberhofer

Carrera C et al. Validity and Reliability of Dermoscopic Criteria Used to Differentiate Nevi From Melanoma. JAMA Dermatol. 2016;152:798-806 